\title{
La cobertura periodística de la conciliación familiar y laboral en el contexto español ${ }^{1}$
}

\section{News coverage of work and family balance in the Spanish context}

Marta Martín Llaguno. Universidad de Alicante

Alejandra Hernández Ruiz. Universidad de Alicante

Marina Beléndez Vázquez. Universidad Alicante

Recibido: 21-XI-2011-Aceptado: 07-XI-2012

Resumen:

Este estudio explora el tratamiento periodístico del conflicto/balance familiar y laboral en España, desde 1996 hasta finales de 2007 (VI, VII y casi la totalidad de la VIII legislatura), a partir de un análisis de contenido de las 312 noticias de El País, ABCy El Mundo. Se identifica un doble ciclo de noticiabilidad impulsado por la Ley de Conciliación pero, especialmente, por la de Igualdad. El Día de la Mujer Trabajadora y El País son esenciales en el marcaje de la agenda. Desde una relativa consonancia informativa, y con gran influencia de los políticos, el tema se encuadra como una cuestión laboral vinculada al espacio público y a las mujeres.

Palabras clave:

Conciliación; conflicto trabajo-familia; agenda research; igualdad de género; mujer.

Abstract:

This study explores the journalistic treatment of the work and family conflict/balance in Spain from 1996 to the end of 2007 (VI, VII and almost the entire parliamentary VIII) from a content analysis of 312 articles published by El Pais, ABC and El Mundo. The paper describes a dual newsworthiness cycle, driven mainly by the Reconciliation and the Equality Act. The Working Women's Day and El País are essential in setting the agenda. The issue is framed in media as a workplace issue related to public space and women.

Keywords:

Conciliation; work-family conflict; press, content analysis; gender equality; women

1 Este artículo es fruto del proyecto I+D financiado por el Instituto de la Mujer. "La construcción social del problema del conflicto trabajo-familia. Análisis mediático, político y de la realidad", con referencia 117/07, dirigido por Marta Martín Llaguno y llevado a cabo entre 2007 y 2010. 


\section{Introducción}

Las principales declaraciones internacionales en materia de conciliación e igualdad mencionan a los medios de comunicación como herramientas cruciales para la sensibilización, o toma de conciencia, sobre estos temas (European Commission, 2000; European Commission, 2006). Dichas menciones no son baladís. En las sociedades actuales, la comunicación colectiva tiene la capacidad de visibilizar y fomentar el conocimiento público de cuestiones que pueden convertirse en problemas sociales. Pero, además, tiene el poder de definirlas y, en consecuencia, de impulsar (o no) determinaciones para solventarlas (Martín Llaguno, 2000a, 2000b).

Ya en 1972 apuntaba acertadamente Downs que la historia de los problemas sociales es la historia de su ciclo de atención. Pues bien, en 2002 se sugería que, en una sociedad dependiente de los medios (DeFleur y Ball-Rockeach, 1989), la historia de los ciclos de atención es a menudo la historia de los ciclos de noticiabilidad (Martín Llaguno, 2002).

De esta manera, en muchas ocasiones los periodistas detectan situaciones disfuncionales en su fase de pre-problema. A raíz de una serie dramática de acontecimientos y del consenso de los informadores sobre la maldad de las circunstancias, la cuestión aparece con omnipresencia en los medios y la sociedad realiza un descubrimiento alarmante. Se debaten las iniciativas de mejora y se analizan sus pros y sus contras en la fase de toma de conciencia de los costes del avance significativo. A medida que se reflexiona sobre el esfuerzo que exige el poner fin a la situación, se pasa a una fase del decrecimiento gradual del interés. En esta etapa, por aburrimiento, por miedo o por una combinación de ambos, la atención vira y el público acaba desinteresándose por el problema inicial. Al final, la cuestión se remplaza por una nueva, situándose en lo que Downs (1972) ha denominado la etapa del post-problema, en la que, bajo unas circunstancias concretas, los medios pueden llevar a cabo una función de recuerdo (Martín Llaguno, 2000a, 2000b y 2002) con referencias esporádicas al tema (ver Cuadro 1). 
Cuadro 1. Ciclo de atención y ciclo de noticiabilidad en la construcción de problemas sociales

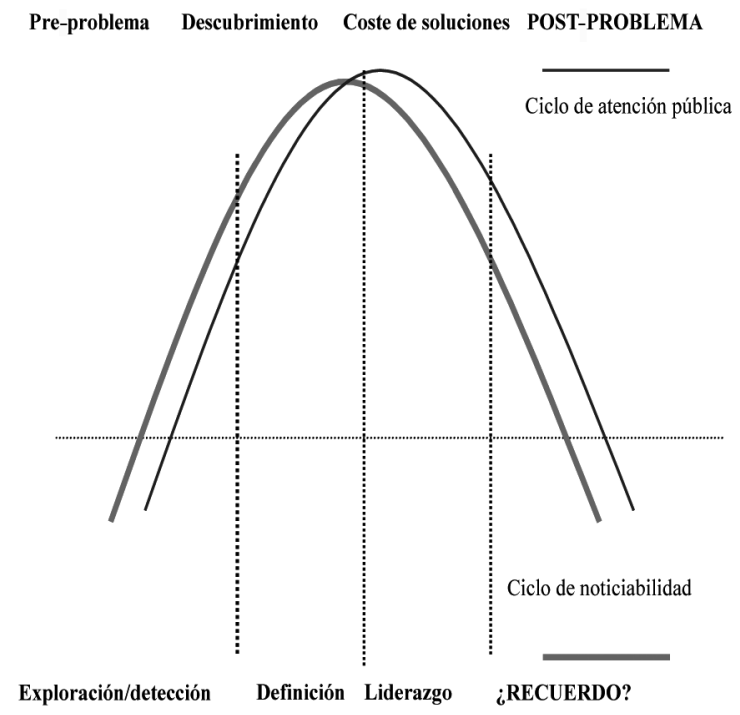

Fuente: Martín Llaguno, M. (2000a, 2000b, 2000c)

El modelo del ciclo de atención (del primer nivel de la agenda, el de las menciones al tema) muestra que la intensidad con la que se realiza la cobertura se relaciona con el grado de relevancia que la sociedad otorga a una cuestión (Martín Llaguno, 2000b; Vives Cases et al., 2005; Martín Llaguno, 2010).

Pero, como afirma Sampedro (1996: 165), "más importante que la cantidad de información sobre los temas /.../ resultan los argumentos con los que ciertos temas se introducen en la agenda mediática”. En este sentido, los trabajos sobre el segundo nivel de la agenda-setting han comprobado que los medios de comunicación no sólo son capaces de transferir a la población la importancia relativa de las cuestiones, sino también la importancia relativa de las valoraciones cognoscitivas y afectivas sobre estas. De este modo, otras características de la cobertura (como el ritmo y los enfoques del problema) se relacionan con la definición colectiva del tema. Por mucho que se persiga una supuesta objetividad, ninguna de las fórmulas que los medios utilizan para encuadrar un asunto es neutral.

Así, en primer lugar, las noticias presentan unas características narrativas y formales determinadas, con unos "formatos" que reflejan la importancia que se le concede a una cuestión en la agenda mediática. El género, la dimensión, ubicación en secciones², y otros “accidentes de las informaciones" son lo que Tankard et al. (1991:3) denominan los "mecanismos del

2 Con la ordenación por secciones no sólo se jerarquizan las informaciones sino que se ofrece al receptor una pista del pacto de lectura 
encuadre". Pues bien, estos mecanismos ayudan a conferir más o menos prominencia a un tema de cara a los lectores (Gersh, 1993).

Tampoco son triviales los "marcos cognitivos" concretos con los que los medios perfilan un problema (enfoques e información movilizadora que permite tomar decisiones). Estos esquemas sirven para organizar los posteriores discursos. Además, las noticias comunican mucho más que hechos (McCombs, 1992: 815) y por eso hay que tener en cuenta también su aspecto afectivo, que se refiere a la respuesta emocional del público que podría resultar de la cobertura. Vendría resumida por los juicios y valores emitidos sobre el tema. Finalmente, la procedencia y la autoría de la información constituyen elementos importantes en los pactos del discurso del lector. Los aspectos mencionados han sido operacionalizados en algunos trabajos del segundo nivel de la agenda-setting, y han servido como base en estudios para resumir la cobertura (Ghanem, 1997: 155; Martín Llaguno, 2002) y describir la tipología de informaciones, que se relaciona con los marcos de decodificación que utilizamos los receptores.

Pese a la importancia probada que las informaciones mediáticas tienen en la construcción y reconstrucción de problemas sociales (Ruiz et al., 2004) y la intuición de que la presencia de la conciliación en los medios ha ido creciendo (Fernández y Tobío, 2005), a diferencia de lo que ocurre en la esfera política, no se han realizado hasta la fecha estudios sobre el tratamiento de esta cuestión. Consideramos que es pertinente revisarla, habida cuenta de la influencia que ha podido tener sobre la percepción y el debate público para su solución.

En el contexto anterior, el estudio presente explora el tratamiento periodístico del conflicto/balance familiar y laboral en España desde 1996 hasta 2007 (VI, VII y casi la totalidad de la VIII legislatura parlamentaria) a partir de un análisis de contenido de informaciones en los diarios escritos. Nuestro interés general es describir el ciclo de noticiabilidad (cómo ha aflorado, se ha liderado, se ha planteado su solución y se ha cerrado o no el tema) e identificar la definición del conflicto hecha por los periódicos. De manera más específica:

Por una parte, se explora la atención prestada al problema por la prensa a lo largo del tiempo. Así, se va a presentar:

1. La acumulación global de informaciones, la tendencia y la estacionalidad de la cobertura y los momentos en los que se han producido aludes informativos;

2. La constancia, omnipresencia y consonancia entre medios en el tratamiento del conflicto/balance familiar laboral ${ }^{3}$.

que debe utilizar, y una idea inicial de cuál va a ser la dimensión cognitiva de la información que se le ofrece.

3 En el proceso de la comunicación social existen elementos no mensurables en los estudios de laboratorio o en las investigaciones puntuales, que, sin embargo, dificultan que los receptores puedan evadirse de las influencias mediáticas. La periodicidad de los mensajes emitidos a través de las instituciones informativas da lugar a una "acumulación" de ideas en la mente de los ciudadanos, que no se aprecia en un experimento aislado. Por otra parte, en una sociedad como la actual, los medios son omnipresentes, y en el caso de la radio y la televisión, su consumo no exige un esfuerzo por parte de los receptores, sino que los medios actúan, en muchas ocasio- 
Por otra parte, se pretende analizar los encuadres periodísticos o las definiciones concretas de la conciliación en este periodo (segundo nivel de la agenda o frames). Así, se va a:

1. Describir las características específicas de las noticias sobre conflicto/balance familiar-laboral presentadas por el conjunto de los medios;

2. Sintetizar toda la información anterior, con el fin de establecer la tipología de informaciones sobre el tema; y

3. Una vez identificados los arquetipos de informaciones se valorará en qué medida han estado presentes en una cabecera u otra y en qué medida han sufrido una evolución ${ }^{4}$.

\section{Métodos}

Para cumplir los objetivos expuestos se ha realizado un análisis de contenido de las noticias sobre el conflicto/conciliación familiar y laboral publicadas entre marzo de 1996 y diciembre de 2007, en los 3 diarios de mayor tirada nacional según la Oficina de Justificación de la Difusión: El País, ABCy El Mundo. Se han incluido todas las informaciones publicadas en la edición nacional impresa, cuyo titular o contenido contemplen uno de los siguientes términos: conciliación; igualdad; conflicto familiar; conflicto laboral; balance laboral. La obtención del corpus se ha realizado mediante búsqueda digital en la página web de los periódicos El País, ABCy El Mundo. En total, se obtuvieron 312 noticias: 136 de El País, 67 de $A B C$ y 109 de El Mundo.

Se ha recogido información, entre otras, de las siguientes variables: presencia del tema, año, mes y legislatura de publicación, morfología de la noticia (tamaño, personificación, sección, acompañamiento de ilustraciones y datos, menciones a instituciones y documentos -categorías no excluyentes- y referencias geográficas), voces (fuente informativa principal

nes, de forma anodina bajo el disfraz del entretenimiento. La "omnipresencia" de los medios confiere inevitablemente a los asuntos que en ellos se tratan, gran resonancia en lo público Finalmente, se ha detectado gran homogeneidad o "consonancia” en la presentación de los contenidos mediáticos. Pese a la proliferación de canales informativos, existe una notable restricción en los contenidos, que implica una limitación en la capacidad de selección de los receptores.

4 Para valorar el potencial efecto de la cobertura mediática de un tema sobre la opinión pública los estudios sobre efectos miden casi siempre la "acumulación", es decir, el número total de noticias en un periodo concreto. A esta aproximación añadimos ya hace años la propuesta de cuantificar también otros dos factores del impacto, señalados teóricamente por Noelle-Neumann (1973: 67-112), pero pocas veces tenidos en cuenta:

(1) La omnipresencia, o el grado de aparición simultánea de un tema, o encuadre en el conjunto de los medios;

(2) La consonancia, o la homogeneidad entre los medios en el aumento o disminución de interés hacia un tema o una dimensión en el tiempo.

Acumulación, consonancia y omnipresencia pueden no confluir con un mismo encuadre del problema, sino concentrarse en enfoques diferentes. En el caso del SIDA, en nuestro trabajo se comprobó que los tres factores del impacto, el nivel de los enfoques del tema, fueron en distinta dirección (Martín Llaguno, 2002). 
-categorías excluyentes: varón, mujer, colectivos-, ámbito de pertenencia de la fuente informativa principal-categorías no excluyentes: sanitario, político, jurídico/judicial, periodístico, y sindical-, protagonista de la información y paciente de la información), definición del problema (término conciliación/balance/conflicto, tratamiento del tema-categorías excluyentes: suceso o tema-; marco principal -categorías no excluyentes-, ámbito de ubicación del tema, problematización, asunción de distribución tradicional de roles), tipo de medidas mencionadas en las noticias (variables no excluyentes: permisos maternidad/paternidad, excedencias, flexibilidad horaria, transferencia económica, servicios públicos) y términos vinculados a conciliación (variables no excluyentes: igualdad/corresponsabilidad), así como responsabilidad del tema.

Para la codificación, tal como se hizo en un estudio previo realizado por los autores (Ruiz et al., 2004), se elaboró un manual para codificadores y se entrenó a dos varones y a dos mujeres. Se empezó con una muestra de 100 noticias para comprobar el grado de concordancia entre ellos. La concordancia en la clasificación de las variables estudiadas fue superior al $80 \%$.

Con objeto de analizar la evolución de la cobertura a lo largo del tiempo, efectuamos un análisis de series temporales, basado en la metodología tradicional para estos estudios que, fundamentalmente, se basa en descomponer las series en varias partes: a) tendencia ${ }^{5}$, b) variación estacional o periódica ${ }^{6}$.

Para describir las características específicas de las noticias se realizó primero un estudio descriptivo sobre la frecuencia de aparición de las distintas variables del protocolo de codificación. Con el objetivo de resumir información, se efectuaron análisis factoriales de componentes principales que permitieron obtener los tipos de noticias. Finalmente, cuando se ha querido observar las relaciones entre variables, además de la prueba del X2 (realizada en las tablas de contingencia), se han utilizado los análisis de la varianza, o ANOVAS de un factor, además de correlaciones cruzadas, que son las correlaciones entre dos series temporales. Las correlaciones cruzadas ayudan a identificar las variables que son indicadores precedentes de otras variables al identificar las observaciones de una serie se correlacionan con las observaciones de otra serie para diversos retardos y adelantos. En el caso concreto de este estudio hemos utilizado esta metodología para identificar las interinfluencias en el tiempo de unas coberturas sobre otras.

Para la realización de los análisis se utilizó el programa SPSS-18.

5 La tendencia es la dirección general de la variable en el periodo de observación, es decir el cambio a largo plazo de la media de la serie. En nuestro caso se ha utilizado para observar la dirección de la cobertura en el tiempo (ascendente o descendente). Para una mejor visualización de los datos nos hemos servido de la técnica del suavizado de medias móviles, que permite limar las fluctuaciones de los valores, con la finalidad de dar una interpretación más compacta de la situación. La media móvil simplemente es una suavización de la tendencia para un seguimiento más claro. Elimina la pronunciación de los dientes de sierra.

6 La estacionalidad corresponde a fluctuaciones periódicas de la variable, en periodos relativamente cortos. Para saber si el tema de la conciliación familiar y laboral es un problema puntual o cíclico en los medios y para detectar cuáles son los factores externos que producen o incitan su cobertura hemos utilizamos este análisis. 


\section{Resultados y discusión}

\subsection{Ciclos informativos, o atención prestada al problema por la prensa a lo largo del tiempo}

El conflicto/la conciliación familiar y laboral no ha sido un tema ni dominante ni estable en la prensa en los últimos doce años. Durante los 140 meses comprendidos entre marzo de 1996 y noviembre de 2007, los diarios analizados publicaron un total de 312 informaciones -lo que supone una media mensual (en el sumatorio de los tres periódicos) de 2 textos informativos. En 46 de los meses no apareció ninguna información sobre el tema, mientras que en algunos periodos se produjo una acumulación de hasta 20 textos.

Pese a esta discreción e inestabilidad inicial, en general, la presencia del conflicto/la conciliación familiar y laboral en $E l$ País, El Mundo y ABC entre 1996 y 2007 fue creciente, como se puede observar en el Cuadro 2 (Ver Cuadro 2. Tendencia conjunta de noticias sobre conciliación y conflicto familiar y laboral de El País, ABC y El Mundo a lo largo de las legislaturas). Así, en la VI legislatura se publicaron 56 noticias sobre el tema, en la VII, 87, y en la VIII 169 noticias.

Cuadro 2. Tendencia conjunta de noticias sobre conciliación y conflicto familiar y laboral de El País (n=136), $A B C(\mathrm{n}=67)$ y El Mundo $(\mathrm{n}=109)$ en las tres legislaturas

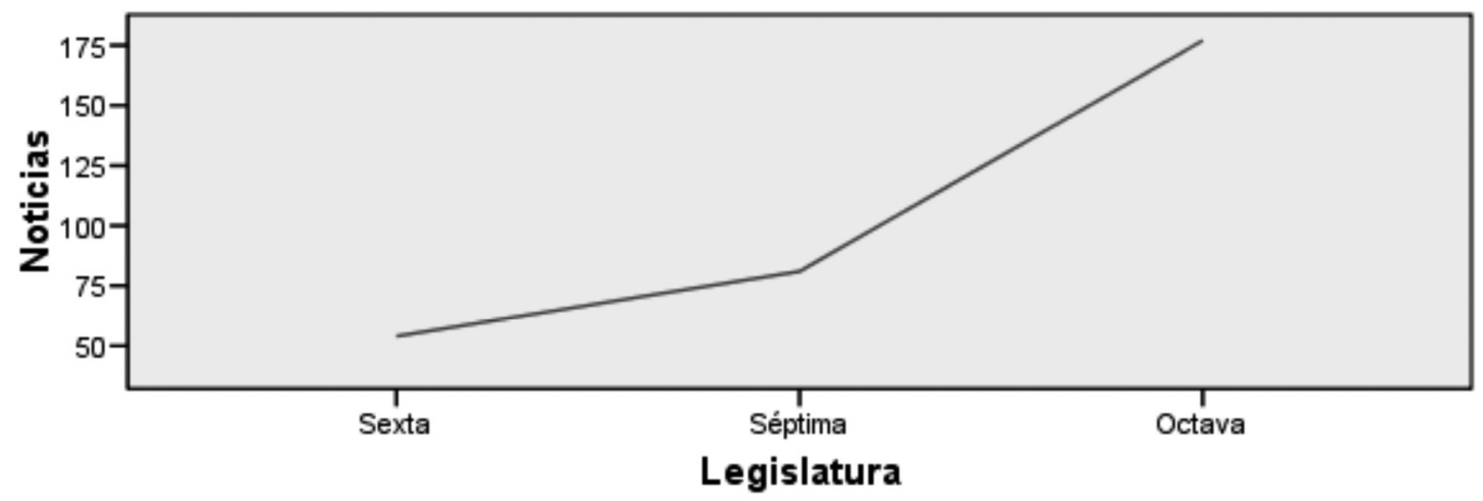

Fuente: Elaboración propia

El Cuadro 3 muestra la distribución mensual conjunta de las noticias con las tendencias (ver Cuadro 3). 
Cuadro 3. Distribución mensual conjunta de noticias sobre conciliación y conflicto familiar y laboral de El País (n=136), ABC (n=67) y El Mundo (n=109)

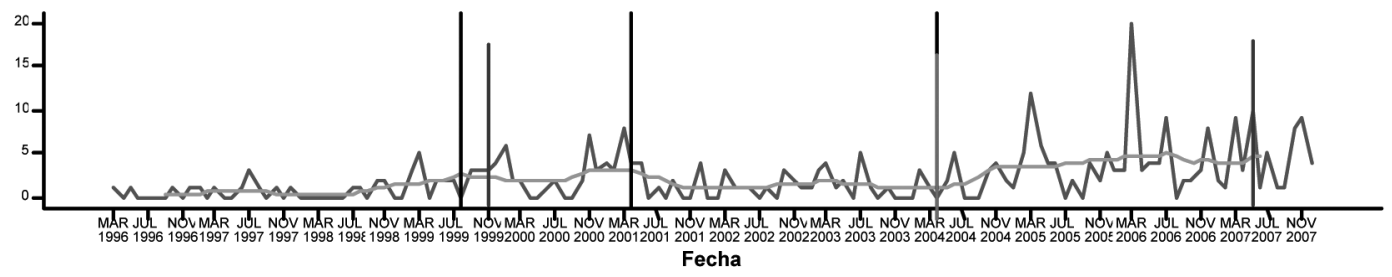

Fuente: Elaboración propia

A lo largo de nuestras tres legislaturas se pueden distinguir dos ciclos de noticiabilidad:

Uno primero, generado por la promulgación de la Ley 39/1999, de 5 de noviembre, para promover la conciliación de la vida familiar y laboral de las personas trabajadoras, que comienza vinculado a la primera legislatura del PP y se cierra en abril de 2004 -con el cambio de gobierno-. En este ciclo, con una cobertura muy baja, se distingue un periodo inicial (desde marzo de 1996 a agosto de 1999) en el que las noticias van ascendiendo; una etapa secundaria, desde esta fecha hasta marzo de 2001, donde se mantienen; y un tercer periodo, hasta abril de 2004, donde la cobertura de nuevo casi desaparece.

Aunque desde 1996, puntualmente, hay informaciones anecdóticas en las que se menciona la conciliación, es en marzo de 1999 cuando se empieza a hablar con asiduidad del tema. A partir de febrero de ese mismo año se presentan en las Cortes las líneas básicas del anteproyecto de ley de conciliación de la vida familiar y laboral que está preparando el Ministerio de Trabajo y Asuntos Sociales (convertido en proyecto de Ley en mayo de 1999). Desde esa fecha hasta noviembre se publican noticias relacionadas con las implicaciones que va a suponer y con las acciones relacionadas con ellas (como el lanzamiento de una campaña publicitaria para animar a los hombres españoles a tomar parte del permiso de maternidad, en vez de sus mujeres, promovida por el Instituto de la Mujer y el Instituto Nacional de Empleo). En noviembre de 1999, cuando la norma es publicada en BOE, se informa sobre sus peculiaridades. La campaña electoral de 2000 hace que la conciliación se presente vinculada a los programas electorales. Sobre todo PP y PSOE mencionan en ellos la necesidad de incrementar las escuelas infantiles y los diarios recogen tímidamente estos planteamientos. Pero, es en 2001, al plantear los partidos de manera explícita políticas familiares, cuando los medios retoman el tema. El Plan Integral de Apoyo a la Familia del PP, de carácter nacional (2001-2004), acapara la atención de los periodistas. Posteriormente, el anuncio de un proyecto de ley del gobierno catalán, con una inversión de 3.000 millones para la conciliación de la vida familiar y laboral de sus funcionarios autonómicos y municipales, también centra el interés periodístico a partir de septiembre de 2001. Finalmente, la presentación de la propuesta de Políticas para el Bienestar de 
las Familias del PSOE, en 2002, mantiene ligeramente las referencias en prensa. Después, estas descienden radicalmente y se cierra el primer ciclo de atención.

Uno segundo, generado con el cambio de gobierno (marzo 2004) y vinculado a la Ley para la igualdad efectiva de mujeres y hombres (LOIEMH), aprobada el 22 de marzo de 2007, que cuenta con las cotas más altas de cobertura del conflicto y que alcanza su periodo álgido a finales de 2007.

En este ciclo, el previsible debate sobre la modificación de la Ley de conciliación en el parlamento anima el primer pico cronológico (segundo en volumen) (n=12) en marzo de 2005. En vísperas del Día Internacional de la Mujer, el gobierno de Rodríguez Zapatero aprueba 54 medidas para avanzar hacia la igualdad plena de las mujeres. Sobre la conciliación de la vida privada y laboral, el titular de Trabajo, Jesús Caldera, anuncia que las administraciones públicas ofrecerán, previa negociación con los sindicatos, un modelo de jornada específica a tiempo parcial para mujeres u hombres con hijos a su cargo. Además, Caldera revela que se crearán escuelas infantiles en los establecimientos militares. El anuncio de acuerdo entre gobierno y sindicatos para el "Plan Concilia", en diciembre de 2005 y la publicación en ese periodo del informe "España en hora europea”, resultado de la ponencia para la racionalización de los horarios en el Congreso, hace subir también la atención.

Pero es la aprobación del Consejo de Ministros del anteproyecto de la Ley para la igualdad efectiva de mujeres y hombres (LOIEMH) el hecho que provoca, en marzo de 2006, la cobertura más extensiva del tema (n=20), en especial en el diario $E l$ País. En el marco de esta acción legislativa, gobierno, oposición, comunidades autónomas y ciudadanos particulares exponen sus posturas sobre un documento que "obliga a las Pymes a negociar planes de igualdad" y que considera a los empresarios como "actores decisivos en el tema”. La oposición considera que esta política va contra la libertad, al igual que la CEOE, mientras que los sindicatos creen que las medidas son insuficientes. Los permisos de paternidad recogidos en este documento y los horarios flexibles tienen el mayor impacto mediático.

Finalmente, en mayo de 2007, la campaña electoral propicia de nuevo que la conciliación sea mencionada en los programas electorales y cubierta por los medios $(\mathrm{n}=10)$. Especial atención recibe el anuncio por parte de Jordi Sevilla del futuro Real Decreto que regula el teletrabajo y que permite que los funcionarios de la Administración General del Estado (AGE) que se acojan a esta posibilidad puedan efectuar telemáticamente la mitad de su jornada

A la luz de lo expuesto, la acumulación de informaciones sobre la conciliación se ha producido de manera mayoritaria en la VIII legislatura, vinculada a la ley socialista de igualdad.

Orden PRE/525/2005 responde al acuerdo llegado en el Consejo de Ministros para adoptar medidas con el propósito de favorecer la igualdad entre mujeres y hombres. En el mismo parecen acciones agrupadas en torno a seis grandes líneas: Empleo, Empresa, Conciliación de la vida familiar y la vida laboral, Investigación, Solidaridad y Deporte. La orden anuncia un séptimo grupo general de medidas de igualdad. Informe de implementación de las 54 medidas para favorecer la igualdad entre mujeres y hombres aprobadas por Consejo de Ministros el 4 de marzo de 2005 publicado mediante la orden de PRE525/2005, de 7 de marzo. 
Sin embargo, a lo largo de todos los periodos, y de los dos ciclos de noticiabilidad, la cobertura tuvo un componente estacional: el conflicto familia-trabajo presentó un patrón cíclico en el tiempo con picos reiterados en marzo (ver Cuadro 4) y descensos en el mes de agosto 8 .

Cuadro 4. Gráfico de la componente estacional estimada

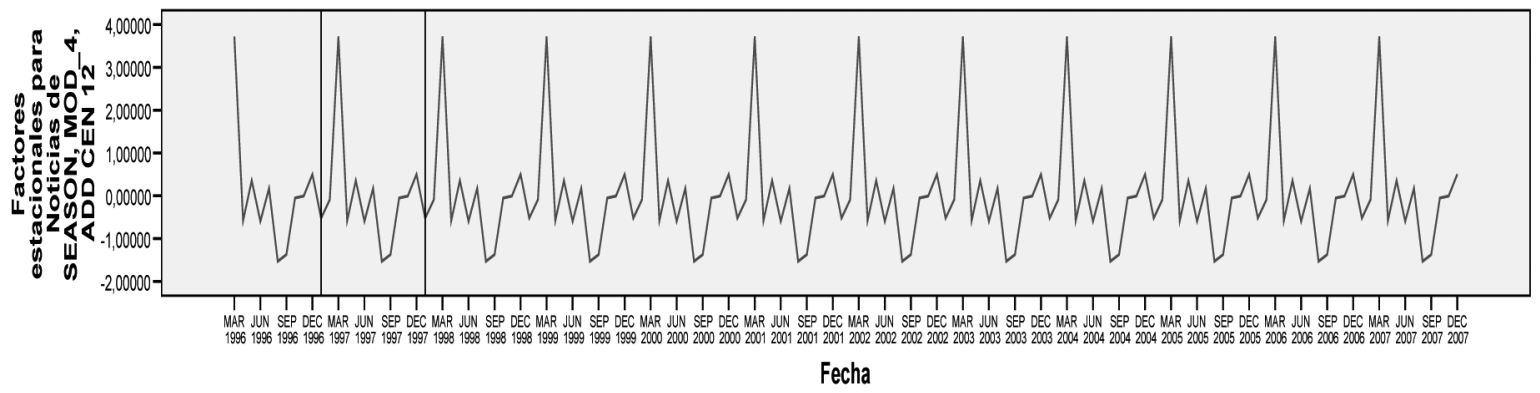

Fuente: Elaboración propia

De alguna manera hay un evento exógeno a los medios que ha "marcado" la cobertura, con independencia de los gobiernos y de las leyes: el día de la Mujer Trabajadora. Esta onomástica parece convertirse desde el principio en un elemento que los periódicos han tomado para ejercer una "función de recuerdo del tema" (Martín Llaguno, 2002). Este hecho pone de manifiesto una relación mediática explícita entre la conciliación y el empleo de la mujer.

\subsubsection{Diferencias e influencias entre diarios}

Analizando los diarios separadamente, aunque la atención prestada a la conciliación difiere entre cabeceras (donde más noticias se publican es en El País, seguido de El Mundo y de $A B C$ ), la acumulación de referencias se produce de manera paralela en El Mundo y en $A B C$ y sigue una tendencia diferente en El País, que acumula textos sobre el tema a más velocidad que los otros medios, especialmente en la última legislatura (ver Cuadro 5).

8 Las funciones de autocorrelación y de autocorrelación parcial corroboran esta estacionalidad, siendo significativas para el retardo 12 con un nivel de confianza del $95 \%$, tomando valores $\mathrm{r}_{12}=0,368$ y $\mathrm{r}_{12}=0,300$, respectivamente. 
Cuadro 5. Acumulación de noticias sobre conciliación y conflicto en las tres legislaturas según diario

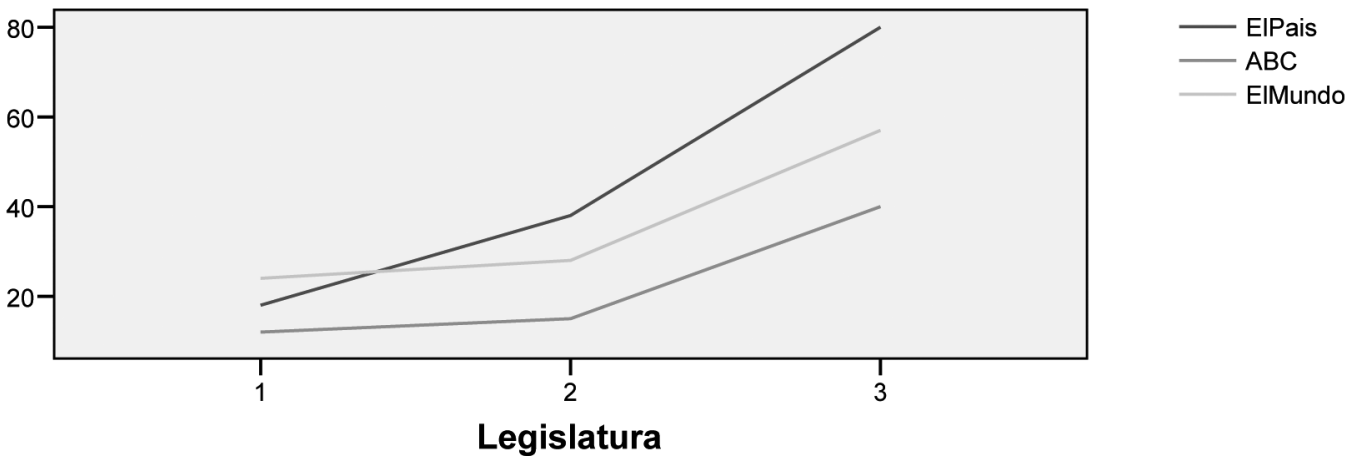

Fuente: Elaboración propia

El Cuadro 6 muestra la distribución del número de frecuencias de noticias a lo largo de de los 142 meses para los tres diarios y el Cuadro 7, las tendencias a partir de un gráfico realizado con la técnica de suavizado mediante medias móviles.
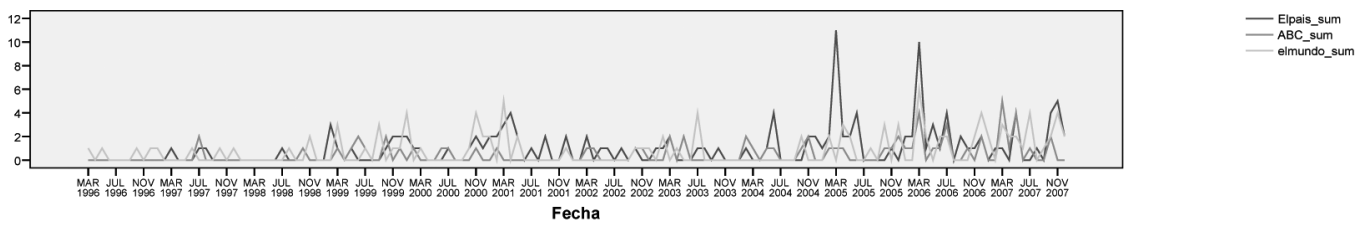

Cuadro 6. Gráfico de secuencias de los tres diarios a lo largo de los 140 meses en estudio

Fuente: Elaboración propia

Cuadro 7. Gráfico de tendencias de los tres diarios a lo largo de los 140 meses en estudio

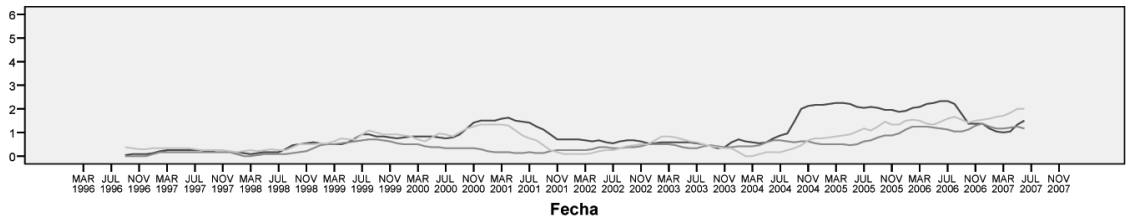

Fuente: Elaboración propia

Como se puede apreciar, y ratificando lo expuesto, El País y El Mundo tienen las mismas tendencias y un volumen parecido de noticias hasta abril de 2004 -periodo inmediatamente posterior al cambio de gobierno-. En ese momento se 
produce un aumento de informaciones en El País que se distancia de los otros dos diarios hasta octubre-noviembre de 2006, cuando El Mundo, tras la promulgación de la Ley de igualdad, inicia un movimiento creciente.

No obstante, la evolución diacrónica de la atención al problema entre diarios se correlaciona significativamente, lo que quiere decir que ha existido una consonancia entre los medios. Sin embargo, los coeficientes obtenidos son menores que los hallados en estudios similares con otros temas (Rogers et al., 1991, obtuvieron correlaciones de hasta 0,91 en un estudio similar sobre la prensa americana entre 1981-1988, el primer ciclo informativo del problema). Parece pues que las fuerzas centrípetas (de las que habla Reese, 1991) que han homogeneizado la cobertura se relacionan con el Día de la Mujer Trabajadora, aunque son menores que las que se producen con otros temas como el SIDA (Martín Llaguno, 2000c).

En efecto, como muestra el Cuadro 8, en todos los diarios hay un aumento de noticias en marzo (y, en menor medida en mayo) que va descendiendo hasta septiembre donde alcanza su nivel mínimo. Estos resultados quedan parcialmente corroborados al realizar el análisis de las autocorrelaciones totales y parciales para cada diario ${ }^{9}$.

En definitiva podemos decir, por tanto, que marzo, con el día de la Mujer Trabajadora, se erige como el factor exógeno que marca la cobertura de los medios en cuestión de conciliación. En menor medida, mayo, con el Día del Trabajador, también es una onomástica importante para el tratamiento periodístico del problema (ver Cuadro 8).

9 En el caso de El País, la función de autocorrelación $\left(\mathrm{r}_{12}=0,291\right)$ y de autocorrelación parcial $\left(\mathrm{r}_{12}=0,230\right)$ aparecen significativas ( $\left.\mathrm{p}<000\right)$ y fuera de los intervalos de confianza $(-0,160 ; 0,160)$ y $(-0,168 ; 0,168)$ respectivamente para el retardo 12 , lo que nos confirma la estacionalidad que apuntaba la representación gráfica de la componente estacional del diario El País, con un aumento de las noticias en marzo y un descenso en septiembre. En el caso de $A B C$, la función de autocorrelación nos da como significativos y fuera del intervalo de confianza los retardos 2, 5 y 12 aunque la función de autocorrelación parcial nos confirma como significativos y fuera del intervalo de confianza los retardos $2\left(\mathrm{r}_{2}=0,19\right)$ y $12\left(\mathrm{r}_{12}=0,20\right)$, lo cual nos puede llevar a pensar en una estacionalidad con periodo 12 y otra con periodo de dos meses.

Pero en el caso de El Mundo la función de autocorrelación nos da como significativo y fuera de los intervalos de confianza únicamente el retardo $4\left(r_{12}=0,23\right)(-0,164 ; 0,164)$ lo que nos avisa de la existencia de una componente estacional con periodicidad de cuatro meses. Para el diario El Mundo no podemos decir que exista un aumento del volumen de noticias para el mes de marzo tan claro como para los otros dos diarios. 
Cuadro 8. Gráfico de la componente estacional estimada. Diario El País, ABCy El Mundo
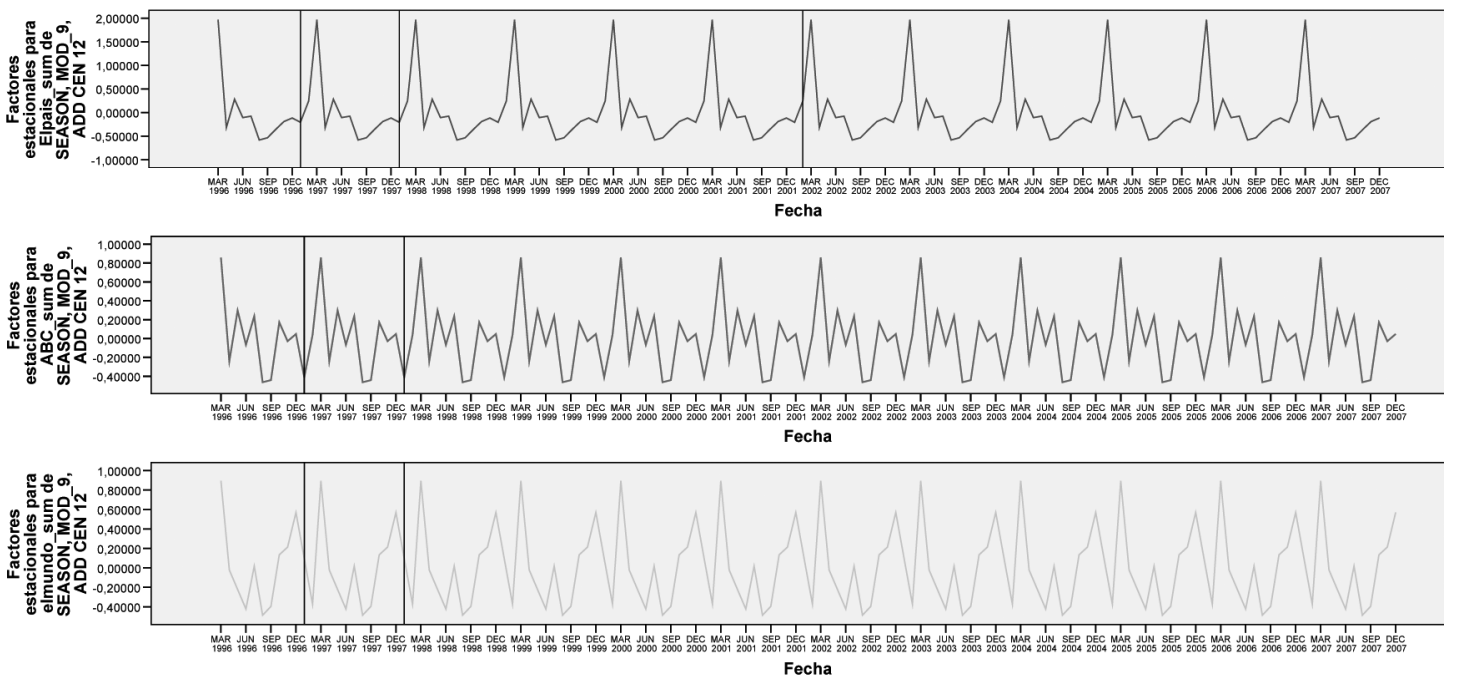

Fuente: Elaboración propia

Aunque la cobertura ha sido pautada más por factores previsibles que por sucesos, como lo demuestran las interinfluencias entre diarios, que han sido débiles en tiempos cortos, y más fuertes en periodos más largos ${ }^{10}$, se podría decir en función de los datos que el medio que más ha marcado la agenda en este caso ha sido El País ${ }^{11}$, (ver Cuadro 9).

10 En efecto, al realizar las correlaciones cruzadas diarias vemos que los valores más altos aparecen en el retardo 0 en el caso de $A B C$ con El País (r=0,1; p<0,015) y en el mismo tiempo en el caso de El Mundo con El País (r=0,09; p<0,015).

11 Las correlaciones cruzadas entre cabeceras con una medida temporal mensual nos indican que la influencia más alta se produce entre El País y $A B C$ en el retardo -12 ( $\mathrm{r}=0,49 ; \mathrm{p}<0,088)$ y en el retardo $0(\mathrm{r}=0,41 ; \mathrm{p}<0,084)$ y en el retardo 9 ( $\mathrm{r}=0,23 ; \mathrm{p}<0,084)$ lo que indicaría que habría influencias de El País sobre $A B C$ aunque también las habría a la inversa con cuatro y cinco meses de retardo (r=0,21; $<<0,084$ y r=0,19; p<0,084). También habría más influencias de El País sobre El Mundo (r=0,3; p<0,084) en el mes inmediatamente posterior que a la inversa $(\mathrm{r}=0,21 ; \mathrm{p}<0,084)$ y de la cobertura de El País sobre los nueve meses posteriores de la de El Mundo (r=0,23; p<0,084). 
Cuadro 9. Correlaciones cruzadas entre diarios

\begin{tabular}{|l|l|}
\hline Mismo mes: 0,339 & \\
\hline Un mes: 0,29 & Un mes: 0,21 \\
\hline Nueve meses: 0,23 & Once meses: 0,14 \\
\hline El País sobre $A B C$ & ABC sobre País \\
\hline Mismo mes: 0,41 & \\
\hline Doce meses: 0,49 & Cinco meses: 0,19 \\
\hline Nueve meses: 0,19 & Cuarto meses: 0,21 \\
\hline
\end{tabular}

Fuente: Elaboración propia

\subsection{Los encuadres periodísticos de la conciliación: las características de las noticias}

El problema de la conciliación familiar y laboral ha sido cubierto mayoritariamente en forma de noticia (68\%) larga (43\%) y en las secciones de Nacional (23\%), Regional (21\%) o Sociedad (13\%). Casi ninguna información se ha acompañado de ilustraciones (31\%) o apoyo numérico (46\%). La cuestión se ha tratado como un tema de cariz atemporal (59\%) más que como sucesos puntuales (40\%). En general, y a diferencia de lo que sucede con otros aspectos relacionados con la igualdad de género como la violencia (Vives et al., 2005), los diarios han reconstruido este tema desde su inicio como un problema social con entidad propia más que como un conjunto de marcos episódicos concatenados entre sí.

La información ha tenido en general un encuadre nacional. Así, un 66,7\% de los textos ha procedido de fuentes con referencias geográficas de este tipo y el 58\% mencionó algún referente español para localizar el acontecimiento o el tema. En cuanto a las instituciones, en el $46 \%$ de los casos aparece el gobierno de España, en el 28\% gobiernos autonómicos, en el 18\% la Unión Europea, en el 13\% gobiernos municipales, en el 10\% el Instituto de la Mujer Nacional y en un 10\% Institutos de la Mujer Autonómicos. En esta misma línea, el 35\% de las noticias ha hecho mención a normativa nacional, el 18\% a planes de igualdad nacionales y el 16\% a planes de igualdad autonómicos. Un 10\% refiere informes académicos.

Con respecto a las voces, en un $12 \%$ de ocasiones no se ha podido identificar al autor de la información. De entre las noticias firmadas, los periodistas han rubricado un $74 \%$ del total y apenas se ha hecho uso de agencias (2,2\%), uno de los elementos claves en la homogeneización de los contenidos entre diarios (Cutlip, 1954; Gold y Simmons, 1965; Snider, 1967).

La descripción mediática del problema se ha caracterizado en primer lugar, por haber sido, en general, en positivo, de forma que el término "conciliación/balance" (35\%) ha sido más frecuente que el de "conflicto/problema" (24\%). En se- 
gundo lugar, y con respecto a la delimitación, el conflicto de roles ha sido mencionado prácticamente con la misma frecuencia (34\%) que el de tareas (35\%) y el de tiempos (30\%). En tercer lugar, el problema ha sido perfilado como una cuestión política (30,7\%) y empresarial (29\%) o institucional (20,3\%), más que cultural y social (20\%). Así, en conjunto, la mayoría de noticias ha tenido un marco de referencia "político/legislativo" (52\%) o "económico" (17\%), quedando muy por detrás el encuadre social, médico o psicológico. No en vano, el ámbito sobre el que se centra la cobertura es el público (59\%) frente al privado (40\%). De ahí que los obstáculos se hayan ubicado más en el trabajo (30\%) o el trabajo y la familia (58\%) que en el ámbito doméstico (11\%). La dimensión política del tema ha copado la agenda informativa en buena parte porque los promotores asiduos de información han sido las políticas (45\%), seguidos, muy por detrás de científicas académicas $(10 \%)$, las instituciones feministas (9\%), el tercer sector y sindicatos $(8,6 \%)$ y las empresas (8\%). En este sentido, las mujeres (43\% de los casos $\left.{ }^{12}\right)$ han hablado sobre el tema muy por encima de los hombres (19\%). Sin embargo, los nombres más citados como fuentes fueron el de Jesús Caldera (Ministro de Trabajo y Asuntos Sociales en el Gobierno de España durante la VIII Legislatura) -con 7 menciones-, Teresa Fernández de la Vega (Vicepresidenta del Gobierno Socialista, con 6), la popular Mercedes de la Merced, presidenta de Mujeres en Igualdad y la también popular Concepción Dancausa, ex directora del Instituto de la Mujer y concejala de Familia y Asuntos Sociales en el Ayuntamiento de Madrid en aquel momento y Manuel Pimentel (Ministro de Trabajo y Asuntos Sociales desde el 18 de enero de 1999 hasta el 19 de febrero de 2000). Además de ser las fuentes más asiduas, los políticos también han protagonizado mayoritariamente las noticias (48\%), seguidos de las instituciones feministas (11\%) y las empresas (9\%). Colectivos (43\%) y mujeres (32\%) han protagonizado más del doble de informaciones que los varones (17\%). El Gobierno, Ana Botella, Caldera y el PP son los actores más mencio$\operatorname{nados}^{13}$.

Los mensajes han problematizado a las mujeres, en exclusiva (40\%) o junto con los hombres (44\%). Sólo un $15 \%$ incide en que la conciliación es un problema no necesariamente femenino. El 53\% de los textos ha asumido en sus esquemas la división tradicional de rol de mujer cuidadora o responsable de la familia. Sin embargo, el 14\% de las informaciones han mencionado el término igualdad y el $11 \%$ corresponsabilidad.

En cuanto a los aspectos más tratados, en el $24 \%$ de los casos se menciona el permiso parental; en el $22 \%$, la prestación/suspensión del permiso de maternidad; en el 15\%, la reducción de la jornada o flexibilidad horaria; y en el 11\%, las excedencias. Sólo un 6\% de las informaciones alude a la adopción.

Pese a que más de la mitad de las noticias enmarca la conciliación en el terreno "político y legislativo", sólo el 21\% atribuye a estos agentes su causa, porcentaje muy similar al $20 \%$ de textos que apuntan a la cultura, al $18 \%$ de informaciones que menciona a las empresas e instituciones y superior al $13 \%$ que acusan a comportamientos privados o individuales. Sin

12 En un $18 \%$ de los casos no se pudo identificar el sexo de la fuente principal.

13 Hay poca visibilidad del debate parlamentario y de los expertos. 
embargo, el 27\% de noticias apunta a que las soluciones tienen que ser políticas y legislativas, seguidas de las empresariales y económicas (17\%) y sólo en tercer lugar ligadas a comportamientos individuales (9\%).

La mayor parte de las acciones cubiertas por las informaciones tienen repercusiones sobre la sociedad en general (50,7\%), sobre el ámbito individual-familiar (15,4\%) o sobre las empresas (12,5\%). Cabe, sin embargo, destacar que, en su mayoría (55\%), están dirigidas exclusivamente a mujeres, frente al 2,5\% destinadas a varones. En definitiva, en las noticias sobre conciliación familiar y laboral, las féminas padecieron (55\%) y dijeron (53\%) mucho más que hicieron (32\%).

Finalmente, el optimismo se ve reflejado en la previsión sobre la evolución del problema que deriva de la lectura de los textos: el $51 \%$ de ellos apunta a que va a mejorar.

\subsection{Las dimensiones de la conciliación/el conflicto familiar $\leftrightarrow$ laboral en las informaciones}

Con el fin de tener una visión más holística sobre el tratamiento periodístico de la conciliación, y con objeto de crear una tipología de noticias, una vez realizada la descripción detallada, las variables anteriores se van a ir sintetizando sucesivamente a partir de que los análisis factoriales resuman los "mecanismos de encuadre", los "marcos cognitivos" y la "dimensión afectiva” utilizados por los periódicos para hablar de la cuestión.

\subsubsection{Los mecanismos de encuadre}

El análisis de componentes principales con rotación Varimax de los ítems relacionados con las características formales de las informaciones (tipo de noticia y tamaño) ha resultado en una solución factorial de tres factores $(\mathrm{KMO}=0,45)$ que explican el $81 \%$ de la varianza.

1. El primero, tratamiento habitual, que da cuenta del $42,7 \%$ de la varianza, incluyó las noticias medias con el formato de pirámide invertida;

2. El segundo, tratamiento reportaje, que da cuenta del $20,2 \%$ de la varianza, comprendió las informaciones más completas y de mayor extensión; y

3. El tercero, un tratamiento editorial, que da cuenta del $17,8 \%$ de la varianza, se relacionó con los artículos de opinión y las cartas.

Por otra parte, con relación a la ubicación en distintas secciones, el análisis factorial de componentes principales con rotación Varimax de los ítems relacionados con la inserción de las informaciones en el periódico (en total 22 variables) revirtió en una solución factorial de cinco factores $(\mathrm{KMO}=0,712)$ que explican el $61 \%$ de la varianza y que muestra cómo las informaciones de conciliación se caracterizaron por tener las siguientes dimensiones: 
En el eje territorial:

1. Una dimensión autonómica local, que supone el $27 \%$ de la varianza, y que localizó las informaciones que hicieron referencia a normativas, institutos de la mujer, y planes de igualdad autonómicos en las secciones de regional o local;

2. Una dimensión nacional (13,4\% de la varianza), que se centró en las noticias con referencias a normativas y planes de igualdad nacionales y a la Constitución, que se ubicaron en las secciones con el mismo nombre;

3. Una dimensión internacional, con noticias con referencias a Naciones Unidas y la Conferencia de Pekín ubicadas en esta sección que explican el 8,2\% de la varianza; y

En el eje temático:

4. Una dimensión económica (6,7\% de la varianza) con noticias con referencias a la UE y a sus tratados y localizada en esta sección; y

5. Una dimensión social (6,5\% de la varianza), con referencias al Instituto de la Mujer y ubicada en esta sección.

\subsubsection{Los marcos cognitivos}

Después de la observación de la descriptiva del análisis de contenido, para resumir los marcos cognitivos que los medios dieron a la conciliación, las variables fuente, actor y receptor de la acción (19 en total), fuertemente correlacionadas entre sí, se operacionalizaron en dimensiones que dieran cuenta de su relación.

El resultado fue la extracción de cuatro vectores $(\mathrm{KMO}=0,794)$ que resumen los marcos cognitivos del tema (y que explican el $64 \%$ de la varianza):

1. En el primero (36,5\% de la varianza), identificable con el enfoque político jurídico de la cuestión, en el que fueron los políticos quienes hicieron y dijeron y la sociedad quien padeció;

2. En el segundo (12\% de la varianza), asociado al enfoque individual empresarial, donde las empresas aparecieron diciendo y haciendo y los individuos padeciendo;

3. El tercero (9\% de la varianza), el enfoque sindical, en el que fue el tercer sector quien dijo, hizo y padeció;

4. El cuarto (7\% de la varianza), el enfoque ciudadano, en el que fue la sociedad quien dijo e hizo y los políticos quienes padecieron. 
Con respecto a la información transmitida a la audiencia sobre la definición específica de la conciliación, es decir, la información movilizadora (Kristiansen y Harding, 1984), después de realizado el pertinente análisis factorial con 16 variables $(\mathrm{KMO}=0,868)$, se resumió en tres vectores que explican el $62 \%$ de la varianza:

1. El primero (41,2\% de la varianza), que llamaremos definición balance, que resume la información que delimitó el problema en estos términos, mencionando la corresponsabilidad y problematizando a las empresas públicas y privadas al tiempo que haciendo menciones específicas a las medidas de permisos maternales y paternales y las excedencias;

2. El segundo (9,2\% de la varianza), relacionado con la definición conflicto, en el que el tema se definió específicamente de esta manera, vinculado a la discriminación positiva y la violencia de género y problematizando a los gobiernos y a la mujer;

3. El tercero (11,6\% de la varianza), denominado definición sexismo, en el que se agrupan las noticias que hablaron de cuestiones culturales y sexistas y que problematizaron a la sociedad y al feminismo.

\subsubsection{La dimensión afectiva}

Para encontrar las dimensiones de los juicios de valor emitidos, se transformaron en puntuaciones factoriales tres dimensiones:

1. La primera, el problema empeorará;

2. $\quad$ La segunda, el problema seguirá igual;

3. La tercera, el problema mejorará.

\subsection{Los arquetipos de noticias sobre la conciliación/el conflicto familiar↔laboral}

Los tres bloques de características extraídos mediante el análisis factorial (mecanismos de encuadre, dimensión cognitiva y dimensión afectiva) son distintos planos que definen una misma realidad: la agenda mediática sobre la conciliación a lo largo de 142 meses en los tres diarios de mayor tirada. Sin embargo, sólo la interrelación de estas magnitudes entre sí nos va a permitir tener una imagen más completa.

Para la elaboración de una tipología de las informaciones publicadas en prensa, se ha llevado a cabo un análisis factorial con los vectores extraídos de los análisis parciales $(\mathrm{KMO}=0,584)$. La realización de esta prueba estadística (que ofrece la ventaja de resumirnos lo esencial de los datos, con el inconveniente de perder parte de la información) nos permite tener un panorama general de las distintas dimensiones de las informaciones sobre conciliación presentadas por la prensa. 
A partir de los resultados del análisis estadístico con SPSS, se pueden identificar 4 dimensiones sobre la conciliación que explican el $69 \%$ de la varianza:

- $\quad$ La primera (33,4\% de la varianza) se corresponde con la dimensión jurídico política del problema conformada con informaciones de este tipo centradas en el conflicto, con formato de noticia habitual ubicadas en las secciones de regional o local y con la idea de que el problema empeorará;

- La segunda la dimensión individual/empresarial del tema, compuesta por noticias centradas en la conciliación, con una visión de que el problema mejorará y publicadas en la sección nacional, explica el 15,7\% de la varianza;

- La tercera, que representaría el 10,5\% de la varianza, viene a resumir la dimensión ciudadana del la conciliación, con formato reportaje donde se tratan casos concretos y se menciona la discriminación; y, finalmente;

- La cuarta (con un 8,9\% de la varianza) compila la dimensión sindical de la conciliación, que estaría conformada con informaciones ubicadas en secciones de internacional y acompañada con opiniones y editoriales.

Estas dimensiones de la conciliación pueden haber aparecido equilibradamente en todos los periódicos estudiados, o haber sido más asiduas en unos diarios que en otros. En la medida en la que esto último suceda, la imagen de la conciliación en la prensa española no habrá sido homogénea, sino que se habrá visto condicionada por componentes endógenos del sistema informativo (el modus operandi y la idiosincrasia de cada medio). Es decir, los diarios habrán promovido diferentes reconstrucciones del problema en sus páginas. Por el contrario, en la medida en que las informaciones con unas características determinadas se vean especialmente asociadas a una fecha, sin diferencia entre diarios, se puede decir que la reconstrucción mediática de la conciliación habrá variado entre unos tiempos y otros; es decir, que la imagen informativa del problema habrá dependido de los tiempos (que en este caso se vinculan con los componentes exógenos del ecosistema informativo). La observación de esta dependencia puede revelarnos cómo ha influido la "programación de la realidad", altamente funcional para medios y promotores. Los factores endógenos (los diarios) y exógenos (en este caso, vinculado a los tiempos) no son excluyentes entre sí. Por tanto, no se descarta la posibilidad de que algunos encuadres de la conciliación hayan estado condicionados tanto por el medio como por las fechas.

Para identificar los encuadres vinculados a un diario (por tanto, las dimensiones generales o específicas de la conciliación), y los enfoques dependientes de la fecha, (por tanto, las dimensiones constantes o periódicas de este tema) se ha realizado una comparación de medias de cada uno de los factores que resume un tipo de noticia.

Atendiendo a los resultados de la comparación de medias con el ANOVA sólo hay dos encuadres de la conciliación que han variado dependiendo del diario: la dimensión sindical de la conciliación ( $F=5,459 ; \mathrm{p}<0,005)$ y la dimensión ciudadana de la conciliación $(\mathrm{F}=5,8439 ; \mathrm{p}<0,003)$, con formato reportaje donde se tratan casos concretos y se menciona la discri- 
minación ${ }^{14}$. Estas dimensiones del problema han sido específicas de algunos medios y por tanto han tenido una presencia significativamente heterogénea en las agendas. En efecto, como se muestra en la tabla resumen de comparaciones múltiples (ver Cuadro 10), siguiendo los resultados que nos ha anticipado el ANOVA, y utilizando dos métodos el Scheffé (basado en la F) y el DMS (basado en la t) para evaluar la dirección y el valor de las diferencias, la dimensión sindical ha estado especialmente presente en El País. Por su parte, la dimensión ciudadana ha estado especialmente ausente de $A B C$ (aunque Scheffé no confirma la diferencia entre $A B C$ y El Mundo, pero DMS sí).

Cuadro 10. Resumen comparaciones múltiples de las dimensiones de la conciliación entre diarios

\begin{tabular}{|c|c|c|c|c|}
\hline \multicolumn{5}{|c|}{ Dimensión sindical } \\
\hline Medio i & Medio j & Diferencias de Medias $(\mathrm{i}-\mathrm{j})$ & Dms P-valor & Scheffé P-valor \\
\hline El País & El Mundo & $+0,3359$ & 0,004 & 0,017 \\
\hline El País & $A B C$ & $+0,3363$ & 0,004 & 0,017 \\
\hline \multicolumn{5}{|c|}{ Dimensión ciudadana } \\
\hline Medio i & Medio j & Diferencias de Medias $(\mathrm{i}-\mathrm{j})$ & Dms P-valor & Scheffé P-valor \\
\hline$A B C$ & El País & $-0,3922$ & 0,001 & 0,004 \\
\hline$A B C$ & El Mundo & $-0,2690$ & 0,022 & 0,073 \\
\hline
\end{tabular}

Fuente: Elaboración propia

Por otra parte, y atendiendo los resultados de la comparación de medias por meses con el ANOVA no paramétrico de Kruskal-Wallis, hay variaciones estadísticamente significativas en la aparición de los encuadres jurídico político (X2=42,2; $\mathrm{p}<0,000)$ y empresarial del problema $(\mathrm{X} 2=68,5 ; \mathrm{p}<0,000)$ que se disparan en los años de promulgación de las normativas (1999 y 2006-7) y en el encuadre ciudadano de la conciliación (X2=21,9; $<<0,024)$ que aparece en los años posteriores a la promulgación de leyes sobre el tema.

\section{Conclusiones}

Las cabeceras de prensa españolas han desempeñado el papel "sensibilizador" que les otorgan las principales declaraciones internacionales en materia de conciliación e igualdad. No obstante, el rol anterior ha sido cumplido modestamente.

14 Para la dimensión ciudadana y para la dimensión sindical los valores del estadístico $\mathrm{F}$ ( $\mathrm{F}=5,843 ; \mathrm{F}=5,459)$ llevan asociados una p de 0 ,003 y 0,005 respectivamente. Por lo tanto, rechazamos la hipótesis nula de que las medias entre los distintos diarios sean iguales para estas dimensiones; existe al menos un diario cuya media es distinta a la media del resto de diarios. 
El tema se ha cubierto en el tiempo, aunque no ha despertado gran interés periodístico. Sólo en momentos puntuales, vinculados a onomásticas, la conciliación ha acaparado especial atención. Además de estos eventos, El País y la planificación política de la cobertura han conseguido marcar (relativamente) la agenda. En efecto, la consonancia en los ciclos de atención en el conjunto de los periódicos ha sido baja en comparación con la encontrada en otros estudios semejantes (Reese, 1991; Martín Llaguno, 2002; Vives-Cases et al., 2005), lo que indica que el conflicto/la conciliación (al menos en los términos en los que se ha presentado) responde a menos criterios de noticiabilidad que otras cuestiones (como el SIDA o la violencia). Si el tema ha permanecido sostenidamente presente en los medios es debido a que, como apuntan Tuñez y Guevara (2009), se ha enmarcado dentro de un orden social programado y ha sido protagonizado por un actor de proyección relevante, principalmente en el ámbito de la gestión política.

Los periódicos han ejercido también una "función traductora” del discurso público, volcada en interconectar el mundo personal de los ciudadanos con el enrarecido ámbito del discurso político (Neuman, Just y Crigler, 1992). Así, el conflicto "familiar y laboral", sufrido por personas -hombres y mujeres- concretas en el contexto de sus trabajos y sus hogares, se ha ido transformando mediáticamente en menos de una década en la "conciliación para la igualdad", exigida a las empresas y manejada por los sindicatos, en línea con la definición que la política (y los políticos) han hecho del tema (Martín Llaguno y Guirao, 2012). La trasmutación se inicia en 1999 con la Ley de conciliación y es impulsada especialmente a partir de 2001 desde la esfera legislativa y política. El enfoque periodístico ha sido en casi todo momento el de que la conciliación es una cuestión laboral vinculada al espacio público y a las mujeres. Los medios han presentado a la ciudadanía un problema ligado al mundo público y a la maternidad, cuya repercusión esencial consiste en impedir la contratación total (o el pleno empleo femenino en las mismas condiciones). Corresponde así a los gobiernos y a las empresas (y no a los individuos o a la cultura) solucionar esta cuestión.

Los periódicos se han hecho fiel eco del enfoque promovido por los organismos políticos supranacionales, en concreto por la UE. De hecho, fecha fijas en el calendario, conocidas y previstas por ciudadanos, legisladores, gobernantes y periodistas (el día del trabajo femenino y el día del trabajo) han permitido preparar acciones especialmente diseñadas para la cobertura mediática.

A la luz de los análisis, el tratamiento periodístico de la conciliación parece haberse vinculado mucho más a la voluntad política que a una demanda social (como sucedió, por ejemplo, en el caso de la violencia machista). La prevalencia en los medios de la dimensión jurídica y empresarial, que responden claramente a la planificación exógena de la cobertura, da cuenta de ello. Por otra parte, la dimensión sindical, el enfoque que un medio concreto, El País, decide dar a este tema, pone de manifiesto el papel de "reconstructores" de la realidad que tienen los periódicos.

Para finalizar, cabe preguntarse no tanto por las dimensiones dominantes sino por las menos presentes y las que han ido cayendo en las curvas de la ausencias mediáticas (Tuñez y Guevara, 2009). En concreto el enfoque ciudadano que se hace 
eco de eventos episódicos concretos y que confiere un tinte humano y personal al tema, especialmente presente en el primer ciclo de noticiabilidad, ha ido desapareciendo con el segundo. En la cobertura de un problema especialmente obtrusive para ciudadanos y ciudadanas, que experimentan cada día el dilema de cómo compaginar su esfera personal y laboral llama la atención el distanciamiento de los medios y la ausencia de información movilizadora. Este hecho, junto con la llamativa presencia de la cuestión en los periodos de campaña electoral, hace cuestionarnos si el uso del conflicto familiar y laboral como argumento de marketing político no está impulsando un tratamiento superficial que comporta el riesgo de que la cuestión (aún sin solucionar) sea percibida por la opinión pública como un problema "pasado", ya dicho, sabido u oído.

\section{Bibliografía}

Cutlip, S. M. (1954): “Content and flow of AP news from trunk to TTS to reader”, Journalism Quarterly, vol. 31, pp. 434-446.

DeFleur, M. L.; Ball-Rockeach, S. J. (1989): Theories of Mass Communication. Nueva York: Logman.

Downs, A. (1972): “Up and down with ecology: the issue attention cycle”, Public Interest, vol. 28, pp. 38-50.

European Commission (2000): Council decision 2001/51/EC of 20 December 2000 establishing a programme relating to the community framework strategy on gender equality (2001-2005), Directorate-General for Employment, Social Affairs and Equal Opportunities, Brussels.

European Commission (2006): Roadmap for equality between women and men 2006-2010, [COM (2006), 92 final], EU, Brussels.

Fernández, J. A.; Tobío, C. (2005): Conciliar las responsabilidades familiares y laborales: políticas y prácticas sociales, Documento de trabajo 79/2005. Madrid: Fundación Alternativas.

Gersh, D. (1993): “Inverted pyramid turned upside down”, Editor \& Publisher, May I, 1993.

Ghanem, S. I. (1997): "El segundo nivel de composición de la agenda: la opinión pública y la cobertura del crimen”, Comunicación y Sociedad, vol. X (1), pp. 151-157.

Gold, D.; Simmons, J. L. (1965): “News selections patterns among Iowa dailies”, Public Opinion Quarterly, vol. 29 (3), pp. 425430.

Kristiansen, C. M.; Harding, C. M. (1984): “Mobilization of health behavior by the press in Britain”, Journalism Quarterly, vol. 61 (2), pp. 364-370.

Martín Llaguno, M. (2000a): “De la detección al recuerdo: una historia periodística del sida”, Zer: Revista de Estudios de la Comunicación, vol. 8, pp. 41-66. 
Martín Llaguno, M. (2000b): "Information, action and reaction. The agenda setting process for anorexia and other eating disorders in Spain", Biomed ethics and appearance meeting of beauty and the doctor, EU Project. Disponible en: http://ec.europa.eu/research/biosociety/pdf/bmh4_ct98_3164.pdf [Consultado el 12/08/2011].

- (2000c): “La función de recuerdo de los medios. Las coberturas de 'grandes temas', Comunicación y Sociedad, vol. XIII (1), pp.115-138.

- (2002): La función de recuerdo de los medios de difusión. ¿Qué pasa cuando en los medios parece no pasar nada sobre un tema? Alicante: Universidad de Alicante.

- (2010): "Historia mediática de la anorexia. La construcción inicial del problema a finales de los 90", Icono14: Revista de Comunicación y Nuevas Tecnologías [en línea], Año 8, Vol. Especial, pp. 45-61. Disponible en: http://www.icono14.net/revista/8-esp/04_icono17_martamartin.pdf [Consultado el 12/08/2011].

Martín Llaguno, M.; Guirao Mirón, C. (2012): “El debate parlamentario sobre conciliación familiar y laboral en España: análisis de una década de iniciativas", Alternativas Cuadernos de Trabajo Social, vol. 19, pp. 57-74. Disponible en: http://publicaciones.ua.es/filespubli/pdf/19899971RD15237645.pdf [Consultado el 19/11/2012].

McCombs, M. (1992): “Explorers and surveyors: expanding strategies for agenda-setting research”, Journalism Quarterly, vol. 69 (4), pp. 813-824.

Noelle-Neumann, E. (1973): "Return to the concept of powerful mass media”, Studies of Broadcasting, vol. 12(9), pp. 67-112.

Neuman, W. R; Just, M.R. y Crigler, Ann N. (1992): Common knowledge: news and the construction of political meaning. Chicago: University of Chicago Press.

Orden PRE/525/2005, de 7 de marzo, por la que se da publicidad al Acuerdo de Consejo de Ministros por el que se adoptan medidas para favorecer la igualdad entre mujeres y hombres, $B O E 57$, de 8 de marzo de 2005, pp. 8111-8114.

Reese, S. D. (1991): “Setting the media’s agenda: a power balance perspective”, Communication Yearbook, vol. 14, pp. 309340 .

Rogers, E.; Dearing, J. y Chang, S. (1991): “Aids in the 1980s: the agenda-setting process for a public issue”, Journalism Monographs, No. 126. Columbia, SC: Association for Education in Journalism and Mass Communication.

Ruiz, M. T.; Martín Llaguno, M.; La Parra, D.; Vives, C. y Albaladejo, M. (2004): "El enfoque de género en las noticias de salud”, Gaceta Sanitaria: Órgano Oficial de la Sociedad Española de Salud Pública y Administración Sanitaria, vol. 18 (Suppl. 2), pp. 65-74.

Sampedro, V. F. (1996): Nuevos movimientos sociales, agendas políticas e informativas: el caso de la objeción de conciencia. Madrid: Instituto Juan March de Estudios e Investigaciones.

Snider, P. (1967): “Mr. Gates revisited: a 1966 version of the 1949 case study”, Journalism Quarterly, vol. 44, pp. 419-427. 
Tankard, J.; Handerson, L.; Sillberman, J.; Bliss, K. y Ghanem, S. (1991): Media frames: approaches to conceptualization and measurement. Paper presented at the Association for Education in Journalism and Mass Communication, Boston, MA, August 7-10, 1991.

Túñez, M.; Guevara, M. (2009): “Framing por proximidad como criterio de noticiabilidad: la curva de las ausencias”, Revista Latina de Comunicación Social, vol.12, pp. 1030-1044. Disponible en: http://www.revistalatinacs.org/09/art/ 878_USC/RLCS_art878.pdf [Consultado el 19/11/2012].

Vives-Cases, C.; Ruiz, M. T.; Álvarez-Dardet, C. y Martín Llaguno M. (2005): “Historia reciente de la cobertura periodística de la violencia contra las mujeres en el contexto español (1997-2001)”, Gaceta Sanitaria: Órgano Oficial de la Sociedad Española de Salud Pública y Administración Sanitaria, vol. 19 (1), pp. 22-28. 